Understanding of Object Rotation Between Two and Three Years of Age

Salome Pedrett, Lea Kaspar, and Andrea Frick

University of Fribourg

(C)American Psychological Association, 2020.

This paper is not the copy of record and may not exactly replicate the authoritative document published in the APA journal. Please do not copy or cite without author's permission.

The final article is available, upon publication, at:

Pedrett, S., Kaspar, L., \& Frick, A. (2020). Understanding of object rotation between two and three years of age. Developmental Psychology, 56, 261-274. DOI: 10.1037/dev0000871

Salome Pedrett, Department of Psychology, University of Fribourg, Switzerland; Lea Kaspar, Department of Psychology, University of Fribourg, Switzerland; Andrea Frick, Department of Psychology, University of Fribourg, Switzerland.

This research was supported by the Swiss National Science Foundation, grant \#PP00P1_150486. We wish to thank Alain Chavaillaz for his help with the eye-tracker setup and for helpful comments on previous versions of the manuscript. We also thank Martina Röthlisberger, Clara Perruchoud, Janine Wieczorek, and Dilan Cümen for their help with data collection and coding. Finally, we wish to thank the parents and toddlers who participated in this study.

Correspondence concerning this article should be addressed to Salome Pedrett, Department of Psychology, University of Fribourg, Rue Faucigny 2, 1700 Fribourg, Switzerland. E-mail: salome.pedrett@gmx.ch 


\begin{abstract}
Toddlers' understanding of object rotation was investigated using a multi-method approach. Participants were 44 toddlers between 22 and 38 months of age. In an eye-tracking task, they observed a shape that rotated and disappeared briefly behind an occluder. In an object-fitting task, they rotated wooden blocks and fit them through apertures. Results of the eye-tracking task showed that with increasing age, the toddlers encoded the visible rotation using a more complex eye-movement pattern, increasingly combining tracking movements with gaze shifts to the pivot point. During occlusion, anticipatory looks to the location where the shape would reappear increased with age, whereas looking back to the location where the shape had just disappeared decreased. This suggests that, with increasing age, the toddlers formed a clearer mental representation about the object and its rotational movement. In the object-fitting task, the toddlers succeeded more with increasing age, and also rotated the wooden blocks more often correctly before they tried to insert them. Importantly, these preadjustments correlated with anticipatory eye movements, suggesting that both measures tap the same underlying understanding of object rotation. The findings yield new insights into the relation between tasks using looking times and behavioral measures as dependent variables, and thus may help to clarify performance differences that have previously been observed in studies with infants and young children.
\end{abstract}

Keywords: mental rotation, object fitting, spatial development, object motion, eyetracking, toddlers 
Mental rotation - the ability to imagine a rotational movement of an object in 2- or 3dimensional space - has been thoroughly investigated in adults since the early 1970s (Cooper \& Shepard, 1973; Shepard \& Metzler, 1971). In classic mental rotation tasks, participants are asked to decide whether a rotated image is the same as a comparison image or a mirror reflection. Mental rotation may also be a prerequisite for basic cognitive tasks, such as recognizing a familiar object in an unfamiliar orientation (cf. Tarr \& Pinker, 1989). Developmental research on this topic, however, has faced a challenging dilemma. Whereas recent research suggests that very young infants are able to infer what an object looks like in a different orientation, many children up to the age of 4 or 5 years perform near chance level when using classic mental rotation tasks (Frick, Möhring, \& Newcombe, 2014).

Similar discrepancies between reports of amazing abilities in infants and profound lacks in older children have also been shown with regard to perspective taking abilities, metacognitive knowledge ("Theory of Mind"), and intuitions about the laws of physics (for reviews, see Keen, 2003; Perner \& Roessler, 2012). These paradoxical results have raised questions of how performance relates to competence, and of whether the same kind of knowledge is measured in studies with different age groups that use different tasks. Do the different tasks tap entirely different knowledge systems? Do these discontinuities reflect a Ushaped development of one single ability that temporarily gets lost and is reacquired years later? Or do they rather reflect changes in representational strength or format, such that knowledge becomes more and more accessible and explicit (cf. Frick et al., 2014; Hespos \& Baillargeon, 2008; Karmiloff-Smith, 1994)?

The aim of the present study is to unravel these discrepancies by systematically testing the same toddlers with different tasks and assessing their performance by means of multiple indices. This will increase our understanding of whether infants and young children can form and transform mental representations of objects. In a larger context, getting a better 
idea of how results obtained using different paradigms are related will also be highly relevant for infant research in general. As Keen (2003, p. 79) pointed out: "this paradox must be resolved before a comprehensive theory of early cognitive development can be constructed". The following sections will give an overview of what different research methods have revealed about how the understanding of object rotation develops on different levels of sophistication.

\section{Observation of rotation}

Eye-tracking studies have shown that already in the first months of life, infants can follow a moving object with their eyes (e.g., Rosander \& von Hofsten, 2002; Rütsche, Baumann, Jiang, \& Mojon, 2006). Infants can also track a small object that moves along a circular trajectory but stays in an upright orientation (Gredebäck, von Hofsten, Karlsson, \& Aus, 2005). However, circular tracking seems to be more difficult for infants than tracking linear horizontal or vertical motion (Grönqvist, Gredebäck, \& von Hofsten, 2006). Less is known about how tracking of rotation develops. Rotational motion is characterized by an object that changes its own orientation (e.g., a 'p' that rotates $180^{\circ}$ and then looks like a 'd'). There are several possibilities for how to observe rotational motion. One possibility is to focus on a point on the rotating object at a certain distance to the pivot point and to follow this point as it moves. This could yield valuable information about the circular trajectory of that particular point. Another possibility is to look only at the stationary pivot point. Yet another option is to switch between the pivot point and points on the object, which could be informative with respect to the changes in orientation of the object. To date, it is unclear which possibility toddlers use when observing rotational motion, and whether gaze patterns change with age.

\section{Anticipation of rotation}

Infants not only follow a moving object with their eyes; they have also been shown to 
look predictively to the location where a linearly moving object would reappear after a short occlusion (e.g., Bertenthal, Gredebäck, \& Boyer, 2013; Johnson \& Shuwairi, 2009; van der Meer, van der Weel, \& Lee, 1994; von Hofsten, Kochukhova, \& Rosander, 2007; Woods, Wilcox, Armstrong, \& Alexander, 2010). This suggests that they are capable of forming a mental representation of the objects and its movement. At 9 months of age, infants even anticipate the reappearance of an upright object that moves on a circular trajectory and is briefly hidden by an occluder (Gredebäck, von Hofsten, \& Boudreau, 2002). However, these anticipatory gaze shifts are not yet fully developed, as they still occur less often than in adults (Gredebäck et al., 2002) and become more frequent between 6 and 12 months of age (Gredebäck \& von Hofsten, 2004). It is therefore likely that predictive eye movements develop further during toddlerhood and generalize to more complex types of motion, such as rotational movement.

\section{Mental rotation}

What might constitute an even more sophisticated type of mental representation is the ability to mentally simulate a rotation precisely enough, so that one knows exactly how a shape would look in a new orientation. For example, when a ' $\mathrm{p}$ ' is rotated $90^{\circ}$ clockwise, one might not only expect that the stem will have a horizontal orientation, but the round part of the 'p' should also face downwards rather than upwards. In other words, the internal spatial relations of the shape should be preserved despite the change in orientation. This ability is typically what is tested in mental rotation tasks for older children and adults that require mirror image discrimination (e.g., Kosslyn, 1980; Shepard \& Metzler, 1971).

Between the fifth and sixth birthday, children can solve most mental rotation tasks (Estes, 1998; Foulkes \& Hollifield, 1989; Frick, Daum, Walser, \& Mast, 2009; Frick, Ferrara, \& Newcombe, 2013; Frick, Hansen, \& Newcombe, 2013; Krüger \& Krist, 2009; Marmor, 1975, 1977; Noda, 2010; Quaiser-Pohl, Rohe, \& Amberger, 2010). However, they still have 
difficulties with some tasks (Dean \& Harvey, 1979; Hawes, LeFevre, Xu, \& Bruce, 2015), suggesting that mental rotation is not yet fully developed. Between the fourth and fifth birthday, children reached an overall accuracy of 60 to $80 \%$ in most studies with two response choices (Estes, 1998; Frick, Hansen, et al., 2013; Noda, 2010; Wimmer, Maras, Robinson, \& Thomas, 2016). At disparities between 90 and $135^{\circ}$, the 4 -year-olds solved $60 \%$ of the trials or more correctly (Frick, Hansen, et al., 2013; Marmor, 1977; Noda, 2010). However, a study with three response choices did not find evidence for mental rotation in 4year-olds (Quaiser-Pohl et al., 2010). Below 4 years of age, research is sparse. In studies that reported accuracy for each disparity separately, the children performed near chance at disparities of $60^{\circ}$ to $180^{\circ}$ (Frick, Ferrara, et al., 2013; Frick, Hansen, et al., 2013; Noda, 2010), suggesting that 4 to 4.5 years is about the lower limit for children to succeed in classic forced-choice mental rotation tasks that present mirror images.

On the other hand, mental rotation abilities have also been investigated in infants, using looking time methods (e.g., Frick \& Möhring, 2013; Frick \& Wang, 2014; Hespos \& Rochat, 1997; Möhring \& Frick, 2013; Moore \& Johnson, 2008; Quinn \& Liben, 2008; Rochat \& Hespos, 1996; Schwarzer, Freitag, Buckel, \& Lofruthe, 2012). For example, Möhring and Frick (2013) presented an object in the form of a 'p' or a 'q', which disappeared behind an occluder and reappeared either in its original form or mirror reversed, in an orientation that varied between 0 and $180^{\circ}$. Infants looked longer when the object that reappeared was mirrored than when it was the same as before the occlusion. This suggests that they anticipated how the object would look after the occlusion by means of mental rotation, and that the mirrored object violated this expectation. These and other results from infant research are hard to reconcile with the above-mentioned findings of pronounced difficulties with behavioral mental rotation tasks in 3- to 4-year-olds. Therefore, it is still an open question how mental rotation develops during the first years of life and through the 
preschool years.

\section{Physical rotation}

Another way to gain insight into toddlers' understanding of rotation is to study how they interact with objects. For example, object-fitting tasks can not only yield valuable information on toddlers' action competencies, they can also shed light on whether toddlers understand and know in advance how they need to rotate an object in order to fit it through an aperture. In a study with 14- to 26-month-olds, Örnkloo and von Hofsten (2007) presented elongated wooden blocks varying in shape, which had to be inserted into a box through various holes. A striking result was that with increasing age, the toddlers more often preadjusted the wooden blocks before they tried to insert them, in that they brought them into the correct orientation already before or during the approach to the hole. Similar results have been obtained in other object-fitting studies (Jung, Kahrs, \& Lockman, 2015, 2018; Smith, Street, Jones, \& James, 2014; Street, James, Jones, \& Smith, 2011), for a review see Lockman, Fears, and Jung (2018). Taken together, the findings of more preadjustments with increasing age demonstrate that toddlers increasingly plan ahead and know in advance that and how they have to rotate an object to fit it into an aperture.

\section{The present study}

In the present study, we investigated whether object-fitting abilities are associated with changes in eye movements during visible rotation, anticipatory eye movements, and mental rotation, by presenting an eye-tracking task and an object-fitting task to the same toddlers. In the eye tracking task, toddlers were presented with computer animations of a shape that looked like the letter ' $p$ ', which rotated clockwise and disappeared behind an occluder. Then, the original shape or its mirror version reappeared on the other side of the occluder. Occluder size and velocity of the rotating shape were varied, and thus the duration the shape was fully occluded, to ensure that the results would generalize to different 
situations.

First, we measured toddlers' eye movements during the visible rotation before the occlusion, with the aim to explore how toddlers encoded the visible rotation, without having a specific hypothesis. Second, we measured toddlers' eye movements when the shape had disappeared behind the occluder. We hypothesized that with increasing age, toddlers would show more anticipatory looks to the reappearance side of the occluder, as their representation of the object motion and thus their expectation about where the object would reappear becomes stronger. Conversely, we also expected that with increasing age, toddlers would less often look back to where the object had disappeared. Third, with respect to mental rotation, we hypothesized that, if toddlers were able to mentally rotate the object and thus had a clear expectation of how the object would look after the occlusion, they would respond with prolonged looking times when the mirrored rather than the original shape reappeared, as this would violate their expectation of object constancy. If, however, infants did not form a clear expectation of how the object should look after the hidden rotation, no differences in looking times between mirrored and original shape could be expected.

In the object-fitting task, which was inspired by Örnkloo and von Hofsten (2007), we addressed the question of whether toddlers understand how an object needs to be rotated physically to fit through an aperture. We hypothesized that toddlers would preadjust the wooden blocks more frequently and insert them more often successfully with increasing age. Crucially, based on the assumption that both paradigms tapped the same underlying understanding of object rotation, we hypothesized that measures of both tasks would correlate.

\section{Method}

\section{Participants}

The final sample consisted of 44 healthy and full-term toddlers ( 20 girls, 24 boys) 
between 22 and 38 months of age $(M=2.56$ years, $S D=0.42)$. Age was used as a continuous variable for statistical analyses, but as categorical variable for some data visualizations, with the age groups of 2 years, 2.5 years, and 3 years ( \pm two months).

Data of 5 additional toddlers were excluded from the analyses because they did not want to wear a sticker on the forehead for the eye-tracking, removed it accidentally, or covered it often with the hand. Data of 8 additional toddlers were excluded because they did not pass the eye-tracking inclusion criteria that are described in detail below.

The toddlers were predominantly from middle-class families and lived near or in a small city in Switzerland. They were recruited in day-care centers, courses, parks, or through the website of the lab. When the child was in the right age range, parents were contacted telephonically and informed about the study. The children were tested in German or French. The parents gave informed, written consent prior to the study. After the study, the toddlers received a certificate and a small gift, such as a toy car or a bubble wand. The study followed ethical guidelines and was approved by the Internal Review Board of the University of Fribourg (project; Development of Spatial Thinking, protocol number: 154).

Power analyses were computed in $\mathrm{R}$ (package: $p w r$ ) to check whether a sample size of 44 participants is sufficiently large to test our hypotheses. The main goals of this study were to detect effects of age, which was used as a continuous variable, as well as correlations between different indices. Power analyses showed that with 44 participants, a power of .80 or more can be reached with a correlation of $r>.40$. Thus, the sample size was large enough to detect medium correlations. Another goal was to detect effects of within-participant variables with two levels (e.g., original vs. mirror object). In a general linear model, a power of .80 or more can be reached for medium within-participant effects $(d>=.43)$. Thus, the sample size was also large enough to test our hypotheses with regard to within-participant effects.

\section{Eye-Tracking Task}


Test environment and apparatus. The experiment took place in a dimly lit room. The room was divided into a section for the participants $(170 \times 170 \mathrm{~cm})$ and a section for the experimenter. The participant section was enclosed by a black curtain and contained a chair, a computer screen, an eye-tracking camera with illuminator, and a video camera. The toddlers were seated in front of the computer screen (ASUS, ROG Swift PG278Q, 27", 2560x $1440,100 \mathrm{~Hz}$ ) either on the parent's lap or in a slightly reclined child car seat. In the latter case, the parent was standing behind, outside of the child's viewing range. The distance between the toddler's forehead and both the eye-tracker camera and the participant screen was approximately $60 \mathrm{~cm}$. The participant screen was slightly tilted so that it was perpendicular to the toddler's line of sight.

The experimenter controlled the experiment from behind the curtain. The experiment was run using Matlab software (R2012a) with Psychtoolbox 3.0.9 (Kleiner et al., 2007) and Eyelink Toolbox (Cornelissen, Peters, \& Palmer, 2002) on a PC with Windows 7. An EyeLink 1000 Remote Eye-tracker (SR Research) recorded the toddler's eye movements at a temporal resolution of $500 \mathrm{~Hz}$. A small sticker was attached to the toddler's forehead, so that the gaze position could be computed despite of head movements. The eye-tracking camera with an 890-nm-illuminator was placed below the participant screen. Saccades (rapid, abrupt eye movements) and blinks were detected using Eyelink's standard thresholds (parameter settings: saccade velocity threshold $=30^{\circ} / \mathrm{s}$, saccade acceleration threshold $=8000^{\circ} / \mathrm{s}^{2}$, saccade motion threshold $=0.1^{\circ}$, blink offset verify time $=12 \mathrm{~ms}$ ).

Below the participant screen and adjacent to the eye-tracker camera, there was a second camera - a video camera with infra-red function (Sony, DCR-AX33). It was linked to a control monitor behind the curtain, so that the experimenter could see the toddler. The camera also recorded the video for offline coding of looking time.

Procedure. The parent was thoroughly informed about the study and instructed not to 
talk to the toddler during trials, and to either close the eyes or to look at the back of the toddler's head during trials. As soon as the toddler and the parent had entered the room, an animation with animals started playing on the participant screen. The toddler sat down, and the sticker for the eye-tracking was attached on the toddler's forehead. If necessary, the toddler was distracted from the sticker placement with a small snack. The seat position and the eye-tracking settings were adjusted, the room lighting dimmed, and the curtain around the participant section closed. A five-point calibration with animated stars that changed in size and color was performed. After the calibration, an abstract flower image followed by a short animation with an octopus were shown in the center of the screen. The set-up took approximately three minutes.

Each toddler was presented with two familiarization trials and four test trials in immediate succession. All trials followed the same general procedure: A pulsating and colorchanging star that was accompanied by cheerful music directed the toddler's attention to the center of the screen. As soon as the toddler looked at the star, the experimenter started a computer animation of a rotating shape on a black background (see below). During this animation, the experimenter pressed a computer key whenever the toddler looked at the screen. The trial was terminated (with a short beeping sound) when the key was released for 2 consecutive seconds or a time-out (see below) was reached.

Familiarization trials. The two familiarization trials featured a magenta stimulus in the shape of the letter ' $T$ ' (see Figure 1A). The lower end of the stem was used as the pivot point when rotating the shape. The stem tapered towards the lower end, so that the pivot point was clearly defined. The pivot point was at the center of the screen. The ' $\mathrm{T}$ ' had a height of 9 $\mathrm{cm}$ and a width of $35^{\circ}$ (i.e., the angle between the left side, the lower end, and the right side of the ' $\mathrm{T}$ ' was $35^{\circ}$ ). Thus, when the stimulus rotated $35^{\circ}$ around its foot, it completely changed location. 
The first familiarization trial showed a fully visible rotation. The computer animation began with a short beep. The stimulus was in an upright position $\left(0^{\circ}\right)$ for the first $2 \mathrm{~s}$ and then rotated $310^{\circ}$ clockwise, stopped, and remained visible. Rotation velocity was $60^{\circ} / \mathrm{s}$ for toddlers in the fast-velocity group or $30 \%$ for toddlers in the slow-velocity group. Measurement of looking time began when the stimulus was $40^{\circ}$ away from its end position (for the sake of comparability with occluded trials); the trial was ended when the toddler had looked away for 2 consecutive seconds, or when $10 \mathrm{~s}$ had elapsed.

In the second familiarization trial, an occluder was introduced (see Figure 1A, first row). Again, the computer animation started with a beep. The ' $\mathrm{T}$ ' was presented in upright orientation, remained there for $2 \mathrm{~s}$, and then started rotating. The stimulus then disappeared behind the occluder, which was grey, shaped like a piece of pie, and covered an angle of 117.5 to $227.5^{\circ}$ (medium occluder). During the full occlusion, the stimulus was completely covered, as its stem tapered towards its lower end. After the stimulus had fully reappeared, it rotated 5 more degrees, stopped moving, and remained visible. Measurement of looking time started at the end of the full occlusion, just when the stimulus started reappearing (i.e., $40^{\circ}$ away from the end position). From this timepoint on, the trial ended when the toddler had looked away for 2 consecutive seconds or when $10 \mathrm{~s}$ had elapsed. Familiarization trials were repeated immediately (maximally twice) if the toddler looked at the participant screen less than $75 \%$ of the time, counted from the start of the trial until $2 \mathrm{~s}$ after the end of the full occlusion.

Test trials. The four test trials had the same event structure as the second familiarization trial, except that the time-out was at $30 \mathrm{~s}$ instead of $10 \mathrm{~s}$. In test trials, the rotating shape resembled the letter ' $p$ ', had a height of $9 \mathrm{~cm}$, a width of $35^{\circ}$, and a stem that tapered towards the lower end (see Figure 1A). The color of the 'p' was red, green, blue, and yellow in the trials $1,2,3$, and 4 , respectively. Thus, each toddler saw the colors in the same 
order. As in familiarization trials, velocity was $60 \%$ s in the fast-velocity group and $30 \% \mathrm{~s}$ in the slow-velocity group.

The four trials varied in occluder size (small / big) and the reappearing shape after the occlusion (original / mirror version). The small occluder (see Figure 1A, second row) had the disappearance side at $117.5^{\circ}$ and the reappearance side at $212.5^{\circ}$, thus covering an angle of $95^{\circ}$. In this case, the stimulus rotated $60^{\circ}$ under full occlusion, with a full occlusion duration of $1 \mathrm{~s}$ (fast velocity) or $2 \mathrm{~s}$ (slow velocity). The big occluder (see Figure 1A, third row) had the disappearance side at $117.5^{\circ}$ and the reappearance side at $242.5^{\circ}$ and thus covered an angle of $125^{\circ}$. In this case, the stimulus rotated $90^{\circ}$ under full occlusion, with a full occlusion duration of $1.5 \mathrm{~s}$ (fast velocity) or $3 \mathrm{~s}$ (slow velocity). Each occluder size was shown twice to a participant: once, the shape that reappeared was the same as before the occlusion ('p'), and once, it was mirrored and thus looked like the letter ' $\mathrm{q}$ '. It was counterbalanced between toddlers in which order they saw the two occluder sizes (small-small-big-big or big-bigsmall-small for the test trials 1 to 4 , respectively), and in which order they saw the shapes after the occlusion (different-same-same-different or same-different-different-same). The toddlers were randomly assigned to these orders as well as the velocity conditions while making sure there were about equal numbers per age group and sex. The test trials were never repeated.

Data analysis. After each session, eye-tracking data was inspected for quality. For each single trial, the raw gaze positions were animated together with the stimuli to check whether the eye movements were properly recorded. This also provided initial information on what types of eye movements toddlers exhibited during different parts of the animation. These mainly consisted of smooth pursuit eye movements, saccades between the stimulus and the pivot point, and saccades to the reappearance side of the occluder.

Areas of interest. The visual inspection of single trials had shown that during the 
visible rotation, children looked mostly at the rotating stimulus or the pivot point. During the full occlusion, they looked mostly either at the disappearance side of the occluder, the pivot point, or the reappearance side of the occluder. For this reason, five areas of interests (AOIs) were defined. The scene AOI covered the entire scene and was a circle with a radius of 13 $\mathrm{cm}$, in the center of the screen. This radius was $3 \mathrm{~cm}$ larger than the radius of the occluder, allowing a margin of measurement error of up to $3 \mathrm{~cm}$. The other AOIs all lay within the scene AOI. The pivot AOI was a circle with a radius of $3 \mathrm{~cm}$, also in the center of the screen. The disappearance AOI covered the disappearance side of the occluder and looked like a piece of pie. It had a width of $70^{\circ}$, which was twice the width of the stimulus. It covered only those areas of the occluder that had a distance of $3 \mathrm{~cm}$ to the center of the screen. Thus, it did not overlap with the pivot AOI. The reappearance AOI had an identical shape but covered the reappearance side of the occluder. The $3-\mathrm{cm}$-criterion that defined the radius of the pivot AOI was a conservative choice and ensured that looking at the pivot point with a measurement error of up to $3 \mathrm{~cm}$ could not be misinterpreted as a predictive eye movement to the reappearance AOI. The last AOI was the stimulus AOI. Unlike the other AOIs, the stimulus AOI was not static, but rotated together with the stimulus. It was also shaped like a piece of pie. It had a width of $70^{\circ}$, a radius of $12 \mathrm{~cm}(3 \mathrm{~cm}$ longer than the stimulus size $)$ and did not overlap with the pivot AOI. Since the stimulus AOI rotated together with the stimulus, it could sometimes overlap with the disappearance or reappearance AOI.

Inclusion criteria. To make sure to include only trials in which the toddlers had seen critical parts of the animation, trials were classified as valid or invalid with an algorithm written in Matlab. Since toddlers made smooth pursuit eye movements as well as saccades, inclusion criteria were based on raw gaze positions. To ensure that the inclusion criteria were appropriate, they were not only theoretically motivated, but additionally informed by inspecting the animations of single trials. A trial was valid if it fulfilled the following four 
criteria. (1) The toddler had encoded the stimulus: In a time window from the start of the trial until the start of full occlusion, the toddler's gaze was on the stimulus AOI for at least 1 s. (2) The toddler had seen the rotation: In a time window from the start of rotation until the start of full occlusion, the toddler looked at the stimulus or pivot AOI for at least $33 \%$ of the time. (3) The toddler was attentive directly before the occlusion: In a time window of $1 \mathrm{~s}$ before the beginning of the full occlusion, the toddler looked at least $200 \mathrm{~ms}$ at the stimulus, pivot, or disappearance AOIs. (4) The toddler was attentive after the occlusion: Within $1.5 \mathrm{~s}$ after the end of the full occlusion, the toddler looked at least $200 \mathrm{~ms}$ at the stimulus, pivot, or reappearance AOIs. The time window for this criterion was $0.5 \mathrm{~s}$ longer than for criterion 3 , because we assumed that some attentive toddlers would still be looking at the disappearance AOI when the stimulus started reappearing, and we wanted to give them enough time to switch their gaze to the stimulus, pivot, or reappearance AOI. A toddler's data was included if at least three out of the four test trials concurred with the inclusion criteria. The 8 children who were excluded from the analyses did not pass the inclusion criteria due to looking away or to the parent, changes in head position, half-closed eyes, or pointing at the screen with the hand. For 37 out of the 44 included children, all four trials were valid.

Calibration accuracy. The average calibration error for the included toddlers was 0.82 visual degrees. This corresponded to an average horizontal error of $0.58 \mathrm{~cm}(S D=0.26)$ and an average vertical error of $0.60 \mathrm{~cm}(S D=0.27)$, which can be considered small with respect to the sizes of the AOIs. The horizontal error did not correlate with age $(r=.09, p=$ $.55)$, nor did the vertical error $(r=.07, p=.65)$.

\section{Wooden Block Task}

Stimuli and apparatus. After the eye-tracking task, the toddlers took a short break and then participated in the wooden block task, which took place in a bright room. The toddlers were seated at a rectangular table, either on a chair or on the parent's lap. The 
experimenter sat at the left side of the table from the child's point of view. Two video cameras filmed the experiment: the main camera was located opposite to the participant; the second camera was placed at the right side of the table. Stimuli were stored beneath the table, outside of the child's view. The parents were instructed to not help the toddlers, but they could encourage and praise the toddlers.

The stimuli consisted of 16 wooden blocks, which were elongated objects that varied in length and in the shapes of their bases (Figure 1C). The term 'base' can be defined as the side of the object that is perpendicular to its longest extension; for example, in a cylinder, the base is a circle. The wooden blocks had to be inserted vertically into a box through apertures that corresponded in shape to the bases of the blocks (Figure 1D). A cylinder, for example, had to be inserted through an aperture that had the shape of a circle.

The wooden blocks varied on four difficulty levels. Two blocks were of very easy difficulty: they had a circular base, with a diameter of $3.4 \mathrm{~cm}$; one was $6 \mathrm{~cm}$ and the other 9 cm long. Two blocks with square base $(2.9 \times 2.9 \mathrm{~cm})$ were considered easy; one was $6 \mathrm{~cm}$ and the other $9 \mathrm{~cm}$ long. The medium difficulty level consisted of 6 blocks, with 3 bases (oval, rectangle, hexagon) and 2 lengths $(6$ or $9 \mathrm{~cm}$ ). These bases were mirror-symmetrical along two axes. Thus, when the blocks were upright, they could be inserted in two ways into the corresponding aperture: the base could have a disparity of either 0 or $180^{\circ}$ with respect to the aperture. The hard difficulty level consisted of 6 wooden blocks, with 3 bases (semicircle, isosceles triangle, isosceles trapezoid) and 2 lengths $(6$ or $9 \mathrm{~cm})$. The bases of these blocks had mirror-symmetry along only one axis, and thus the blocks could be inserted in only one way when upright: the base had to have a disparity of $0^{\circ}$ with respect to the aperture. The bases of medium and hard blocks measured roughly $4.2 \times 2.3 \mathrm{~cm}$.

The wooden box in which the blocks had to be inserted was $19.8 \mathrm{~cm}$ wide and deep, and $13.8 \mathrm{~cm}$ high. The inside was covered in black foam rubber to soften the impact when the 
blocks were jolted into the box. A large aperture on the left side allowed the experimenter to remove the blocks after a trial. Exchangeable lids could be placed on the top, each with an aperture in its center that corresponded to the base of a wooden block. The lids were made of plywood and covered with beige foam rubber. The lids were oriented such that the blocks would fit if oriented as depicted in Figure 1C from the child's point of view. The box was approximately $15 \mathrm{~cm}$ below the child's eye-level, so that the child could clearly see the shape of the apertures. The wooden blocks were presented on a black cuboid, which was placed behind the box, from the toddler's viewpoint. It had the same width as the box, but was $5 \mathrm{~mm}$ higher and $11.5 \mathrm{~cm}$ deep.

Procedure. Each toddler was presented with the same order of trials (see Table 1): 2 training trials (easy), 6 test trials (medium and hard), 2 baseline trials (very easy), and another 6 test trials (medium and hard). At the start of the experiment, the experimenter explained to the toddler that the game was about sticking blocks into the box, demonstrated with a training stimulus how to insert it into the aperture, and then let the toddler insert both training stimuli. After the toddler had successfully inserted both training stimuli, the test trials began.

At the beginning of each trial, the experimenter placed the lid on the box and the wooden block on the pedestal in one of two possible orientations (see Table 1). The orientations had in common that the block was placed flat, diagonally on the pedestal (Figure 1D). In one orientation, the block's longitudinal axis extended from close left to far right from the toddler's viewpoint $\left(+45^{\circ}\right)$, and in the other orientation, it extended from close right to far left $\left(-45^{\circ}\right)$. The experimenter held the wooden block slightly at the far end and asked the toddler: "How does this one fit through the hole?" The experimenter then released the block, so that the toddler could grasp it. If the toddler successfully inserted the block into the aperture (see also criteria for success below), the experimenter praised the toddler and confirmed: "Yes, that's the way it fits through the hole!" If necessary, the toddlers were 
asked to try again, and if they did not succeed within $20 \mathrm{~s}$ after touching the block for the first time, the experimenter helped inserting the block. This ensured that each toddler was provided with the same feedback regardless of success. After each trial, the experimenter removed the block and lid.

\section{Results}

The eye-tracking data were analyzed based on raw gaze positions (without saccades or blinks), because the toddlers not only looked at static parts of the scene such as the pivot point or the occluder, but also often tracked the rotating stimulus with slow eye movements (smooth pursuit). In addition to raw gaze positions, saccades were analyzed in order to count how often the toddlers switched between AOIs. A switch was defined as a gaze shift between two AOIs with at least one saccade in between.

\section{General visual attention}

On average, the 44 toddlers looked at the scene AOI in $90 \%$ of the time, measured from the start of the animation until 1.5 seconds after the end of the full occlusion. The remaining $10 \%$ consisted of saccades, blinks, looking away, or other kinds of data loss. An ANCOVA showed that age (mean-centered covariate) and velocity (fast / slow) had no main effects on the percentage of time the toddlers looked at the scene AOI (both $F_{\mathrm{s}}<1$ ). Age interacted with velocity, $F(1,40)=4.93, p=.03, \eta_{\mathrm{p}}{ }^{2}=.11$. Post-hoc linear regressions showed that age effects went in opposite directions in the two velocity conditions, but both effects were not significant (in the fast-velocity condition: $b=7.08, p=.08$; in the slowvelocity condition: $b=-4.81, p=.18)$.

\section{Observation of rotation}

To explore how the toddlers observed the rotating shape, the data during the fully visible rotation before the occlusion were analyzed. The toddlers looked mostly at the stimulus AOI, but also attended the pivot point (see Figure 2). In the fast velocity condition, 
they looked in $69 \%$ of the time at the stimulus AOI $(S D=17)$ and in $20 \%$ of the time at the pivot AOI $(S D=15)$. Similarly, in the slow velocity condition, they looked in $64 \%$ of the time at the stimulus AOI $(S D=16)$ and in $20 \%$ of the time at the pivot $\mathrm{AOI}(S D=14)$.

The toddlers also switched between looking at the stimulus AOI and the pivot AOI. In the fast velocity condition, they switched 0.53 times per trial $(S D=0.33)$ from the pivot to the stimulus AOI, and 0.27 times per trial $(S D=0.26)$ from the stimulus to the pivot AOI. In the slow velocity condition, they switched 0.8 times per trial $(S D=0.5)$ from the pivot to the stimulus AOI, and 0.65 times per trial $(S D=0.48)$ from the stimulus to the pivot AOI.

Next, we explored how these four measures were related among each other. All intercorrelations were controlled for velocity. Looking at the stimulus AOI was negatively correlated to looking at the pivot AOI $(r=-.79, p<.001)$. Looking at the stimulus AOI was also negatively correlated to switches from the stimulus to the pivot AOI $(r=-.45, p=.002)$ and to switches in the other direction $(r=-.46, p=.002)$. Looking at the pivot AOI, switches from the stimulus to the pivot AOI, and switches from the pivot to the stimulus AOI were all positively intercorrelated (all $r \mathrm{~s}>.49$, all $p \mathrm{~s}<.001$ ). These correlations suggested a trade-off between following the stimulus AOI versus looking at the pivot AOI and switching to and from the pivot AOI. Visual inspection of the data confirmed that some children looked almost exclusively to the stimulus AOI, whereas some children distributed their gaze about equally between the stimulus and the pivot AOI. Most children were somewhere in between these extremes and showed a combination of mostly tracking the stimulus but also attending to the pivot point. No toddler looked exclusively at the pivot point, as all toddlers looked at the stimulus $\mathrm{AOI}$ in at least $33 \%$ of the time.

Possible age-related changes in how the toddlers observed the visible rotation were analyzed by means of ANCOVAs, with velocity (fast / slow) as between-participants variable and age as mean-centered covariate. Separate ANCOVAs were performed for each of the 
four dependent variables separately. A first analysis revealed that with increasing age, the toddlers looked less long at the stimulus AOI, $F(1,40)=4.51, p=.04, \eta_{\mathrm{p}}^{2}=.10$. Neither the main effect of velocity nor the interaction term was significant (both $F_{\mathrm{S}}<1$ ). A second analysis showed that with increasing age, the children switched more often from the stimulus to the pivot AOI, $F(1,40)=4.57, p=.04, \eta_{\mathrm{p}}{ }^{2}=.10$. Velocity had a significant main effect, $F(1,40)=11.36, p=.002, \eta_{\mathrm{p}}{ }^{2}=.22$, but did not interact with age $(F<1)$. A third ANCOVA showed that neither age nor velocity had an effect on the percentage of the time the toddlers looked at the pivot AOI, all $F_{\mathrm{S}}(1,40)<=1.10$, all $p \mathrm{~s}>=.30$. A fourth ANCOVA showed that age did not influence how often the toddlers switched from the pivot to the stimulus AOI ( $F$ $<1$ ), but that switches in this direction were more frequent in the slow than the fast velocity condition, $F(1,40)=4.44, p=.04, \eta_{\mathrm{p}}^{2}=.10$, without interacting with age $(F<1)$. To summarize, the toddlers looked significantly less long at the stimulus AOI and switched more often from the stimulus to the pivot AOI with increasing age, see Figure 3A and 3B.

\section{Anticipation of rotation}

Figure 2 suggests that with increasing age, the toddlers looked more anticipatorily to the reappearance AOI. To test this statistically, arrival times were computed, using a time window that consisted of the full occlusion plus $1.5 \mathrm{~s}$, in order to include the data of toddlers who only shifted their gaze in response to the reappearing stimulus. Time zero was defined as the time when the full occlusion ended; negative values indicated anticipatory eye movements. For example, an arrival time of $-0.5 \mathrm{~s}$ would indicate that gaze arrived at the reappearance AOI half a second before the stimulus started reappearing. Arrival time could be computed for all trials that had passed the inclusion criteria, except for two trials in which the gaze never arrived at the reappearance AOI in the given time window. These two trials were excluded.

Developmental changes in arrival times were analyzed with a repeated-measures 
ANCOVA. The independent variables were velocity (between-participants, fast / slow) and occluder size (within-participant, small / big). Age was entered as a covariate (continuous and mean-centered). Velocity as well as occluder size had significant main effects on arrival time: toddlers' gaze arrived more in advance when the velocity was slow compared to fast, $F(1,40)$ $=29.70, p<.001, \eta_{\mathrm{p}}^{2}=.43$, and when the occluder size was big compared to small, $F(1,40)$ $=13.58, p<.001, \eta_{\mathrm{p}}^{2}=.25$. Velocity and occluder size did not interact $(F<1)$. As expected, age had a significant effect on arrival time (see Figure 3C): with increasing age, gaze arrived earlier at the reappearance AOI, $F(1,40)=8.55, p=.006, \eta_{\mathrm{p}}{ }^{2}=.18$. The age effect was similar in all conditions, as there were no significant interactions with velocity and/or occluder size (all $F \mathrm{~s}<2.24$, all $p \mathrm{~s}>.13$ ). Table 2 illustrates that the 2-year-olds' gaze arrived at the reappearance AOI approximately when the stimulus started reappearing if occlusion duration was $1 \mathrm{~s}$, but clearly in anticipation of the stimulus if occlusion duration was $3 \mathrm{~s}$. The 3-year-olds showed clear anticipatory eye movements in all conditions.

To test whether age differences in anticipatory eye movements were not just due to development in speed, but that younger children less clearly expected the stimulus to reappear at the reappearance side, we tested whether younger children looked back to the disappearance side more often. Looking back was defined as a switch (see above) to the disappearance AOI, from either the pivot AOI, the reappearance AOI, or the area of the occluder between the disappearance and reappearance AOI. Because the toddlers rarely looked back in the fast-velocity condition, in which occlusion duration was likely too short to look back, only the data of the slow-velocity condition were analyzed. On average, the toddlers looked back 0.36 times per trial $(S D=0.31)$. Linear regression revealed that with increasing age, the toddlers looked back significantly less often $(b=-0.47, p=.003$; see Figure 3D). Figure 2 also shows that looks to the disappearance AOI near the end of the occlusion phase decrease with age. 


\section{Mental rotation}

As an indicator for mental rotation, looking time was analyzed. Looking time was defined as the cumulative time of looking at the scene AOI after the end of the full occlusion, before looking away for 2 consecutive seconds or the time-out was reached. Looking time was coded manually in Datavyu Software (Datavyu Team, 2014). To assess reliability, looking time was also computed using the raw eye-tracking gaze positions, which showed a strong correlation with the manually coded looking times $(r=.95)$.

To test whether the toddlers looked longer at the scene when the shape that reappeared was the mirror image rather than the original shape, looking times were analyzed with a multilevel model ( $R$-package: afex; function: mixed). The fixed effects were the shape after the occlusion (within-participant, original / mirror image), occluder size (withinparticipant, small / big), velocity (between-participants, fast / slow), and age (continuous and mean-centered). The model included random intercepts. Results showed that looking times were similar when the shape after the occlusion was the original $(M=8.66 \mathrm{~s}, S D=5.53)$ and when it was the mirror image $(M=7.74 \mathrm{~s}, S D=5.16)$, and there were no other significant main effects or interactions (all $F \mathrm{~s}<1.64$, all $p \mathrm{~s}>.19$ ). Looking times did thus not provide evidence for mental rotation in the tested age range.

\section{Wooden block task}

For the wooden block task, we report the data of the 44 children who were included in the eye-tracking task. For these children, all trials of the wooden block task were analyzed. Videos were coded using the Datavyu software. The start of a trial was defined as the point in time when the toddler touched the wooden block the first time.

Preadjustment. To test whether the toddlers knew in advance how they needed to rotate the wooden blocks before trying to fit them through the apertures, preadjustments were analyzed. Analysis of preadjustment was based on the point in time when the wooden block 
was both upright and placed near or on the aperture for the first time. Being upright was defined as deviating less than $30^{\circ}$ from vertical. To code whether the block was placed near or on the aperture, a rectangular AOI on the box's lid was defined, which covered the aperture and a margin: each of the AOI's four sides was located at a third of the distance between the border of the aperture and the side of the box. A wooden block was considered as placed near or on the aperture when a part of it touched the lid within the AOI or - if directly over the hole - had the same altitude as the lid's surface. Inter-rater reliability for determining the point in time when the block was for the first time both upright and placed near or on the aperture was excellent $(r=.97)$. At this timepoint, a wooden block was considered preadjusted if the orientation of its base differed maximally $30^{\circ}$ from the aperture's orientation. This could be coded for all trials, except for one in which the block was never both upright and placed near or on the aperture. This trial was coded as not preadjusted. Two independent raters agreed in $97 \%$ of the trials whether the block was preadjusted or not.

A repeated-measures ANCOVA on preadjustment was calculated, with difficulty (medium / hard) as within-participant variable and age as covariate (continuous and meancentered). The analysis revealed a significant effect of difficulty, $F(1,42)=41.13, p<.001$, $\eta_{\mathrm{p}}{ }^{2}=.49$ (Figure $\left.4 \mathrm{~A}\right)$. The toddlers preadjusted the medium blocks in $76 \%$ of the trials $(S D=$ 23 ), and the hard blocks in $43 \%$ of the trials $(S D=27)$. (This effect was expected, as blocks of medium difficulty could be preadjusted in two ways once upright, whereas blocks of hard difficulty could be preadjusted in only one way). Preadjustment increased significantly with age, $F(1,42)=9.41, p=.004, \eta_{\mathrm{p}}^{2}=.18$. Age and difficulty did not interact, $F(1,42)=1.47, p$ $=.23$.

Success. A trial was coded as successful if the wooden block was inserted at least 1 $\mathrm{cm}$ into the aperture within the available $20 \mathrm{~s}$. Two independent raters agreed on $100 \%$ of the 
trials. Because success rate was not normally distributed and had unequal variances across difficulty levels and age, it was analyzed using non-parametric statistics. Overall, the toddlers were highly successful. At medium difficulty, all toddlers had a success rate of $100 \%$, except for one toddler who solved all but one trial successfully. At hard difficulty, the average success rate was $83 \%(S D=24)$. The difference between medium and hard difficulty was significant (Wilcoxon signed-rank test: $Z=3.75, p<.001$ ). Because the toddlers solved almost all trials of medium difficulty successfully, age effects were investigated at the hard difficulty level (Figure 4D). Toddlers solved significantly more hard trials successfully as age increased (Spearman's rho $=.59, p<.001)$.

For additional information, the time of success was also coded (inter-rater reliability: $r=.98$; see Figure 4B). Age differences could already be observed early in the trial, and the likelihood of solving the task within the first $4 \mathrm{~s}$ increased with age (medium difficulty: Spearman's rho $=.44, p=.003$; hard difficulty: Spearman's rho $=.33, p=.03$; see Figure 4C).

\section{Correlations among performance measures}

To test the hypothesis that predictive eye-movements are related to object-fitting performance, we computed correlations between arrival time in the eye-tracking task and preadjustment as well as success in the object-fitting task. Since the success rate had considerable variance at hard difficulty only, success at hard difficulty was used for correlations. Results showed that there was a significant negative correlation between arrival time in the eye-tracking task and preadjustment in the wooden block task, if controlled for velocity $(r=-.48, p=.001)$ or velocity, age, and how much the children attended to the scene AOI during visible rotation $(r=-.37, p=.018)$. The more frequently toddlers preadjusted the objects in the wooden block task, the earlier they looked to the reappearance AOI in the eyetracking task (Figure 5). There was no correlation between arrival time in the eye-tracking 
task and success at hard difficulty in the wooden block task, Spearman's rho $=-.14, p=.38$.

Finally, we explored all other possible correlations of measures within and between tasks. For the eye-tracking task, these were the four measures of how the children encoded the visible rotation (looking at stimulus AOI, looking at pivot AOI, switches from stimulus to pivot AOI, switches from pivot to stimulus AOI) as well as arrival time, looking back to the disappearance AOI, and looking-time differences between the mirror object and the original object. As mentioned above, the four measures of how the toddlers encoded the visible rotation were significantly intercorrelated. In addition, results showed that the more the toddlers had switched from the stimulus to the pivot AOI during the visible rotation, the earlier their gaze arrived at the reappearance AOI, controlled for velocity $(r=-.48, p=.001)$. This correlation remained significant if controlled not only for velocity, but also for age and how much the children looked at the scene AOI during the visible rotation $(r=-.38, p=.01)$. Switches in the other direction, from the pivot AOI to the stimulus, during the visible rotation were also related to earlier arrival times, controlled for velocity $(r=-.51, p<.001)$. This also remained significant if controlled for velocity, age, and how much the toddlers looked at the scene AOI during the visible rotation $(r=-.46, p=.002)$. In addition, there was a nonsignificant trend for earlier arrival time the less toddlers looked at the stimulus AOI during the visible rotation, controlled for velocity $(r=.27, p=.08)$. However, arrival time was not correlated with how much the toddlers looked at the pivot AOI during the visible rotation, controlled for velocity $(r=-.23, p=.14)$.

Within the wooden block task, preadjustment and success rates at hard difficulty were significantly correlated (Spearman's rho $=.53, p<.001$ ), even if controlled for age (rho $=$ $.42, p=.005)$. The more often toddlers preadjusted the blocks of hard difficulty, the more often they succeeded at inserting them. There were no other correlations within or between tasks (all $|r \mathrm{~s}|<.30$, all $p \mathrm{~s}>.14$ ). 


\section{Additional analyses}

Each of the above-mentioned performance measures were also analyzed for sex differences. There were no significant main effects of sex on any of these variables.

Moreover, the statistical results of all parametrical tests were verified using permutation tests, which were programmed in R. A permutation test is a resampling approach that allows for computing $p$-values without making any assumptions about the underlying distribution of the data. These permutation tests yielded $p$-values that were very close to the $p$-values reported above, and using this approach did not change whether any of the observed results reached statistical significance (at $p<.05$ ) or not.

\section{Discussion}

In this study, we investigated toddlers' understanding of rotational object movement. We measured how they observe a rotating shape, whether they anticipate an observed rotational movement, whether they know in advance how they need to rotate an object to fit it through an aperture, and whether they can mentally rotate an object. Overall, the results showed marked changes in several of these skills between 2 and 3 years of age, suggesting pronounced developmental progression in toddlers' cognition about object rotation. Moreover, switches between the stimulus and the pivot point during visible rotation were associated with anticipatory looking, which in turn was related to toddlers' preadjustments of wooden blocks in the object fitting task, suggesting a tight relation between visual processing of rotational object movement and action competencies.

\section{Observation of rotation}

The eye-tracking results during the visible rotation showed that with increasing age, the toddlers looked less exclusively to the stimulus and switched more often from the stimulus to the pivot point. Thus, rotational motion evoked more complex eye-movement patterns than previously observed with upright objects moving on a circular trajectory (e.g., 
Gredebäck et al., 2005). Since motion attracts attention (e.g., Franconeri \& Simons, 2003), looking less exclusively at the rotating stimulus might involve an active disengagement process. With increasing age, toddlers might learn to inhibit looking at moving parts of a scene, so that they can more flexibly attend other relevant features. This may provide them with more detailed information on how the shape changes its orientation over time.

\section{Anticipation of rotation}

The result that the toddlers looked more anticipatorily to where the rotating object was likely to reappear after the occlusion extends previous findings (e.g., Gredebäck et al., 2002) by indicating that anticipatory eye movements continue to develop during toddlerhood. The finding that the toddlers less frequently looked back to the disappearance AOI with increasing age suggests that anticipatory eye movements did not simply get faster with age, but that the toddlers developed a stronger expectation that the object would reappear at the reappearance side rather than the disappearance side of the occluder.

\section{Mental rotation}

The present study also tested mental rotation using a violation-of-expectation paradigm - a looking-time method that has previously provided evidence for mental rotation in 6- to 10-month-old infants (e.g., Frick \& Möhring, 2013; Möhring \& Frick, 2013). In the present study, however, looking times did not yield evidence for mental rotation in toddlers, and there were no age effects nor correlations of looking-time differences with other dependent variables. This result can be explained in at least three different ways. A first possibility is that toddlers are not capable of mental rotation. A second possibility is that toddlers can perform a mental rotation, but that they did not do it in the present task - maybe because they did not sufficiently pay attention to whether the shape looked like a 'p' or ' $q$ ', as this was not salient enough or they were busy attending to other features. A third possibility is that the toddlers did perform a mental rotation, but that looking time may not 
have been an appropriate measure to capture this competence in the present task or for the present age group. Similarly, Langer, Gillette, and Arriaga (2003) did not find significant results in 21-month-olds' looking times, which contrasted with their successful performance in a search task - but see also He, Bolz, and Baillargeon (2011) for a successful application of a violation-of-expectation paradigm with 2.5 -year-olds. Looking times can be influenced by a number of factors and reflect various cognitive processes, such as expectations of what will happen on the screen next, or curiosity about what their parent thinks of the event. We therefore cannot draw any conclusions about whether toddlers are capable of mental rotation on the basis of these non-significant looking-time results.

\section{Physical rotation}

In line with previous findings from object-fitting tasks (Jung et al., 2015, 2018; Örnkloo \& von Hofsten, 2007; Smith et al., 2014; Street et al., 2011), the toddlers' preadjustment and success rates in the wooden block task developed considerably between 2 and 3 years of age. This indicates that with age, the toddlers increasingly considered in advance how they needed to rotate the wooden blocks in order to insert them into the apertures.

Preadjustment could be achieved by two different strategies. Using a physical-rotation strategy, the toddler may physically rotate the object (e.g., in the air) and compare it with the aperture. If the comparison indicates that the object is not yet correctly oriented, the steps of rotating and comparing can be repeated, until the comparison indicates that the object is aligned with the aperture. Alternatively, using a mental-rotation strategy, the toddler may rotate the object mentally and then compare this mental image with the aperture. Both strategies have in common that they require a sophisticated understanding of object rotation, as in both strategies, the toddlers consider how the block has to be rotated before they physically try to insert it. Based on our data, we cannot distinguish between these two 
strategies with certainty. However, the mental rotation strategy could be expected to be faster than physically acting out each step. Thus, the increasing number of toddlers who already solved the task within $4 \mathrm{~s}$ might indicate a development from a slower physical-rotation strategy to a faster mental-rotation strategy between 2 and 3 years of age. Future studies using higher-resolution motion tracking technology may further explore these strategies.

\section{Correlation between encoding of visible rotation and arrival time}

In the eye-tracking task, switches between the stimulus and the pivot AOI during the visible rotation (as opposed to extensive tracking of the stimulus) were associated with earlier anticipatory eye movements to the reappearance side of the occluder. This correlation remained significant even if controlled for age, velocity, and how much the toddlers looked at the scene during visible rotation. Thus, general developmental maturation or general visual attention cannot account for the correlation. A precondition for both switches and anticipatory looks is the ability to disengage from looking at one location and to make saccades to another location. Young infants have often been found to stick to an object with their gaze (Hood \& Atkinson, 1993). In toddlerhood, the ability to disengage may still be improving and give rise to individual differences. That is, toddlers who are better able to switch their gaze during visible rotation might also be less likely to stick to the disappearance side of the occluder once the object has disappeared, and more likely to shift their gaze across the occluder. A more specific reason for the correlation between switches during visible rotation and arrival time might be that the switches had enabled the toddlers to understand how the orientation of the object changed as it rotated, leading to a more detailed representation of the object and its movement, and thus to a clearer expectation.

\section{Correlation between arrival time and preadjustment of wooden blocks}

As a main finding, the present study showed a significant correlation between anticipatory looks in the eye-tracking task and preadjustments in the wooden block task. This 
correlation was still significant if controlled for age, velocity, and how much the children looked at the scene AOI during visible rotation. Thus, it is unlikely that general age-related maturation or visual attention had influenced this correlation.

This close relation between anticipatory looks and preadjustment, together with the findings that measures of both tasks underwent considerable development across the investigated age range (i.e., over a period of 16 months), suggests that visual processing of rotational object movement and action competencies develop in parallel. Moreover, the findings of rather sophisticated (but still developing) rotation understanding in toddlerhood render the possibility unlikely that infants are endowed with knowledge that gets lost during toddlerhood and is regained around age 5. The data rather suggest that competencies increase continuously across age.

The correlational findings and the parallel developmental progression thus corroborate the notion that the different measures reflect a common underlying competency. A possible common mechanism underlying predictive eye movements and preadjustments of wooden blocks may be the basic ability to mentally simulate object rotation. In the eyetracking task, mentally simulating the rotational movement during occlusion might have allowed the toddlers to know where the rotating shape was going to reappear. In the wooden block task, mental simulation might have allowed the toddlers to know in advance how the base of the wooden block needed to be rotated to fit through the aperture.

The finding of a parallel and synchronized development in different performance measures is also in line with dynamic systems theory of development (cf. Smith \& Thelen, 2003), which conceptualizes development as the emergent product of continually linked and mutually interactive processes that occur over time. In accordance with this systemic view, our findings suggest that in order to thoroughly understand developmental processes, it is useful to combine different research paradigms and simultaneously assess multiple 
performance measures within the same children. Future studies may also attempt to chart the developmental time course of individual measures within children, using longitudinal designs.

\section{Conclusion}

The present study showed striking developmental parallels between toddlers' eye movements when observing visible rotation or anticipating hidden rotation, and their ability to preadjust wooden blocks and to fit them successfully into apertures. In all of these areas, some competencies were present at age 2 , but they still improved markedly until the age of 3 . A common mechanism underlying predictive eye movements and preadjustments of wooden blocks may be the ability to mentally simulate object rotation. The present findings suggest that toddlerhood is an age when broad changes happen in the understanding of object rotation. 


\section{References}

Bertenthal, B. I., Gredebäck, G., \& Boyer, T. W. (2013). Differential contributions of development and learning to infants' knowledge of object continuity and discontinuity. Child Development, 84, 413-421. doi:10.1111/cdev.12005

Cooper, L. A., \& Shepard, R. N. (1973). The time required to prepare for a rotated stimulus. Memory \& Cognition, 1(3), 246-250. doi:10.3758/BF03198104

Cornelissen, F. W., Peters, E. M., \& Palmer, J. (2002). The Eyelink Toolbox: Eye tracking with MATLAB and the Psychophysics Toolbox. Behavior Research Methods, Instruments, \& Computers, 34, 613-617. doi:10.3758/bf03195489

Datavyu Team. (2014). Datavyu: A Video Coding Tool. Databrary Project, New York University. URL http://datavyu.org/.

Dean, A. L., \& Harvey, W. O. (1979). An information-processing analysis of a Piagetian imagery task. Developmental Psychology, 15, 474-475. doi:10.1037/00121649.15.4.474

Estes, D. (1998). Young children's awareness of their mental activity: The case of mental rotation. Child Development, 69, 1345-1360. doi:10.1111/j.14678624.1998.tb06216.x

Foulkes, D., \& Hollifield, M. (1989). Responses to picture-plane and depth mental-rotation stimuli in children and adults. Bulletin of the Psychonomic Society, 27, 327-330. doi:10.3758/bf03334617

Franconeri, S. L., \& Simons, D. J. (2003). Moving and looming stimuli capture attention. Perception \& Psychophysics, 65, 999-1010. doi:10.3758/bf03194829

Frick, A., Daum, M. M., Walser, S., \& Mast, F. W. (2009). Motor processes in children's mental rotation. Journal of Cognition and Development, 10, 18-40. doi:10.1080/15248370902966719 
Frick, A., Ferrara, K., \& Newcombe, N. S. (2013). Using a touch screen paradigm to assess the development of mental rotation between $3 \frac{1}{2}$ and $5 \frac{1}{2}$ years of age. Cognitive Processing, 14, 117-127. doi:10.1007/s10339-012-0534-0

Frick, A., Hansen, M. A., \& Newcombe, N. S. (2013). Development of mental rotation in 3to 5-year-old children. Cognitive Development, 28, 386-399. doi:10.1016/j.cogdev.2013.06.002

Frick, A., \& Möhring, W. (2013). Mental object rotation and motor development in 8- and 10-month-old infants. Journal of Experimental Child Psychology, 115, 708-720. doi:10.1016/j.jecp.2013.04.001

Frick, A., Möhring, W., \& Newcombe, N. S. (2014). Development of mental transformation abilities. Trends in Cognitive Sciences, 18, 536-542. doi:10.1016/j.tics.2014.05.011

Frick, A., \& Wang, S. H. (2014). Mental spatial transformations in 14- and 16-month-old infants: Effects of action and observational experience. Child Development, 85, 278293. doi:10.1111/cdev.12116

Gredebäck, G., \& von Hofsten, C. (2004). Infants' evolving representations of object motion during occlusion: A longitudinal study of 6- to 12-month-old infants. Infancy, 6, 165184. doi:10.1207/s15327078in0602_2

Gredebäck, G., von Hofsten, C., \& Boudreau, J. P. (2002). Infants’ visual tracking of continuous circular motion under conditions of occlusion and non-occlusion. Infant Behavior and Development, 25, 161-182. doi:10.1016/S0163-6383(02)00119-4

Gredebäck, G., von Hofsten, C., Karlsson, J., \& Aus, K. (2005). The development of twodimensional tracking: A longitudinal study of circular pursuit. Experimental Brain Research, 163, 204-213. doi:10.1007/s00221-004-2162-0

Grönqvist, H., Gredebäck, G., \& von Hofsten, C. (2006). Developmental asymmetries between horizontal and vertical tracking. Vision Research, 46, 1754-1761. 
doi:10.1016/j.visres.2005.11.007

Hawes, Z., LeFevre, J.-A., Xu, C., \& Bruce, C. D. (2015). Mental rotation with tangible three-dimensional objects: A new measure sensitive to developmental differences in 4- to 8-year-old children. Mind, Brain, and Education, 9, 10-18. doi:10.1111/mbe.12051

He, Z., Bolz, M., \& Baillargeon, R. (2011). False-belief understanding i 2.5-year-olds: evidence for violation-of-expectation change-of-location and unexpected-contents tasks. Developmental Science, 14, 292-305.

Hespos, S. J., \& Baillargeon, R. (2008). Young infants' actions reveal their developing knowledge of support variables: converging evidence for violation-of-expectation findings. Cognition, 107, 304-316. doi:10.1016/j.cognition.2007.07.009

Hespos, S. J., \& Rochat, P. (1997). Dynamic mental representation in infancy. Cognition, 64, 153-188. doi:10.1016/S0010-0277(97)00029-2

Hood, B. M., \& Atkinson, J. (1993). Disengaging visual attention in the infant and adult. Infant Behavior and Development, 16, 405-422. doi:10.1016/0163-6383(93)80001-O

Johnson, S. P., \& Shuwairi, S. M. (2009). Learning and memory facilitate predictive tracking in 4-month-olds. Journal of Experimental Child Psychology, 102, 122-130. doi:10.1016/j.jecp.2008.02.004

Jung, W. P., Kahrs, B. A., \& Lockman, J. J. (2015). Manual action, fitting, and spatial planning: Relating objects by young children. Cognition, 134, 128-139. doi:10.1016/j.cognition.2014.09.004

Jung, W. P., Kahrs, B. A., \& Lockman, J. J. (2018). Fitting handled objects into apertures by 17- to 36-month-old children: The dynamics of spatial coordination. Developmental Psychology, 54, 228-239. doi:10.1037/dev0000420

Karmiloff-Smith, A. (1994). Beyond Modularity: A Developmental Perspective on Cognitive 
Science. International Journal of Language \& Communication Disorders, 29, 95-105. doi: $10.3109 / 13682829409041485$

Keen, R. (2003). Representation of Objects and Events: Why Do Infants Look So Smart and Toddlers Look So Dumb? Current Directions in Psychological Science, 12, 79-83. doi:10.1111/1467-8721.01234

Kleiner, M., Brainard, D., Pelli, D., Ingling, A., Murray, R., \& Broussard, C. (2007). What's new in psychtoolbox-3. Perception, 36, 1-16.

Kosslyn, S. M. (1980). Image and mind. Cambridge, MA: Harvard University Press.

Krüger, M., \& Krist, H. (2009). Imagery and motor processes - When are they connected? The mental rotation of body parts in development. Journal of Cognition and Development, 10, 239-261. doi:10.1080/15248370903389341

Langer, J., Gillette, P., \& Arriaga, R. I. (2003). Toddlers' cognition of adding and subtracting objects in action and in perception. Cognitive Development, 18, 233-246. doi:10.1016/S0885-2014(03)00022-4

Lockman, J. J., Fears, N. E., \& Jung, W. P. (2018). The development of object fitting: The dynamics of spatial coordination. Advances in Child Development and Behavior, 55, 31-72. doi:10.1016/bs.acdb.2018.05.001

Marmor, G. S. (1975). Development of kinetic images: When does the child first represent movement in mental images? Cognitive Psychology, 7, 548-559. doi:10.1016/00100285(75)90022-5

Marmor, G. S. (1977). Mental rotation and number conservation: Are they related? Developmental Psychology, 13, 320-325. doi:10.1037/0012-1649.13.4.320

Möhring, W., \& Frick, A. (2013). Touching up mental rotation: Effects of manual experience on 6-month-old infants' mental object rotation. Child Development, 84, 1554-1565. doi:10.1111/cdev.12065 
Moore, D. S., \& Johnson, S. P. (2008). Mental rotation in human infants: A sex difference. Psychological Science, 19, 1063-1066. doi:10.1111/j.1467-9280.2008.02200.x

Noda, M. (2010). Manipulative strategies prepare for mental rotation in young children. European Journal of Developmental Psychology, 7, 746-762. doi:10.1080/17405620903465771

Örnkloo, H., \& von Hofsten, C. (2007). Fitting objects into holes: On the development of spatial cognition skills. Developmental Psychology, 43, 404-416. doi:10.1037/00121649.43.2.404

Perner, J., \& Roessler, J. (2012). From infants' to children's appreciation of belief. Trends in Cognitive Sciences, 16, 519-525. doi:10.1016/j.tics.2012.08.004

Quaiser-Pohl, C., Rohe, A. M., \& Amberger, T. (2010). The solution strategy as an indicator of the developmental stage of preschool children's mental-rotation ability. Journal of Individual Differences, 31, 95-100. doi:10.1027/1614-0001/a000017

Quinn, P. C., \& Liben, L. S. (2008). A sex difference in mental rotation in young infants. Psychological Science, 19, 1067-1070. doi:10.1111/j.1467-9280.2008.02201.x

Rochat, P., \& Hespos, S. J. (1996). Tracking and anticipation of invisible spatial transformations by 4- to 8-month-old infants. Cognitive Development, 11, 3-17. doi:10.1016/S0885-2014(96)90025-8

Rosander, K., \& von Hofsten, C. (2002). Development of gaze tracking of small and large objects. Experimental Brain Research, 146, 257-264. doi:10.1007/s00221-002-1161-2

Rütsche, A., Baumann, A., Jiang, X., \& Mojon, D. S. (2006). Development of visual pursuit in the first 6 years of life. Graefe's Archive for Clinical and Experimental Ophthalmology, 244, 1406-1411. doi:10.1007/s00417-005-0248-4

Schwarzer, G., Freitag, C., Buckel, R., \& Lofruthe, A. (2012). Crawling is associated with mental rotation ability by 9 -month-old infants. Infancy, $18,432-441$. 
doi:10.1111/j.1532-7078.2012.00132.x

Shepard, R. N., \& Metzler, J. (1971). Mental rotation of three-dimensional objects. Science, 171(3972), 701-703. doi:10.1126/science.171.3972.701

Smith, L. B., Street, S., Jones, S. S., \& James, K. H. (2014). Using the axis of elongation to align shapes: Developmental changes between 18 and 24 months of age. Journal of Experimental Child Psychology, 123, 15-35. doi:10.1016/j.jecp.2014.01.009

Smith, L. B., \& Thelen, E. (2003). Development as a dynamic system. Trends in Cognitive Sciences, 7, 343-348. doi:10.1016/S1364-6613(03)00156-6

Street, S. Y., James, K. H., Jones, S. S., \& Smith, L. B. (2011). Vision for action in toddlers: The posting task. Child Development, 82, 2083-2094. doi:10.1111/j.14678624.2011.01655.x

Tarr, M. J., \& Pinker, S. (1989). Mental rotation and orientation-dependence in shape recognition. Cognitive Psychology, 21, 233-282. doi:10.1016/0010-0285(89)90009-1

van der Meer, A. L. H., van der Weel, F. R. R., \& Lee, D. N. (1994). Prospective control in catching by infants. Perception, 23, 287-302. doi:10.1068/p230287

von Hofsten, C., Kochukhova, O., \& Rosander, K. (2007). Predictive tracking over occlusions by 4-month-old infants. Developmental Science, 10, 625-640. doi:10.1111/j.1467-7687.2007.00604.x

Wimmer, M. C., Maras, K. L., Robinson, E. J., \& Thomas, C. (2016). The format of children's mental images: Penetrability of spatial images. European Journal of Developmental Psychology, 13, 582-593. doi:10.1080/17405629.2015.1132623

Woods, R. J., Wilcox, T., Armstrong, J., \& Alexander, G. (2010). Infants' representations of three-dimensional occluded objects. Infant Behavior and Development, 33, 663-671. doi:10.1016/j.infbeh.2010.09.002 
Table 1

Order of the trials in the wooden block task.

\begin{tabular}{lllllll}
\hline $\begin{array}{l}\text { trial } \\
\text { number }\end{array}$ & type & difficulty & base & $\begin{array}{l}\text { length } \\
\text { (cm) }\end{array}$ & color & orientation \\
\hline 1 & training & easy & square & 9 & magenta & \\
2 & training & easy & square & 6 & dark green & \\
3 & test & medium & hexagon & 9 & orange & $-45^{\circ}$ \\
4 & test & hard & semi-circle & 6 & cyan & $-45^{\circ}$ \\
5 & test & medium & oval & 9 & violet & $+45^{\circ}$ \\
6 & test & hard & isosceles trapezoid & 6 & orange & $+45^{\circ}$ \\
7 & test & medium & rectangle & 9 & cyan & $-45^{\circ}$ \\
8 & test & hard & isosceles triangle & 6 & violet & $-45^{\circ}$ \\
9 & baseline & very easy & circle & 9 & yellow & $+45^{\circ}$ \\
10 & baseline & very easy & circle & 6 & dark blue & $-45^{\circ}$ \\
11 & test & hard & isosceles triangle & 9 & green & $+45^{\circ}$ \\
12 & test & medium & rectangle & 6 & red & $+45^{\circ}$ \\
13 & test & hard & isosceles trapezoid & 9 & blue & $-45^{\circ}$ \\
14 & test & medium & oval & 6 & green & $-45^{\circ}$ \\
15 & test & hard & semi-circle & 9 & red & $+45^{\circ}$ \\
16 & test & medium & hexagon & 6 & blue & $+45^{\circ}$ \\
\hline
\end{tabular}


Table 2

Arrival time (seconds) and percentage of anticipatory trials by velocity, occluder size and age group. The duration of the full occlusion (seconds) is determined by velocity and occluder size.

\begin{tabular}{|c|c|c|c|c|c|c|}
\hline \multirow[b]{2}{*}{ velocity } & \multirow[b]{2}{*}{ occluder size } & \multirow[b]{2}{*}{$\begin{array}{l}\text { full } \\
\text { occlusion } \\
\text { duration }\end{array}$} & \multirow[b]{2}{*}{ age group } & \multicolumn{2}{|c|}{ arrival time } & \multirow{2}{*}{$\begin{array}{l}\begin{array}{l}\text { anticipatory } \\
\text { trials }^{1}\end{array} \\
M\end{array}$} \\
\hline & & & & $M$ & $S D$ & \\
\hline \multirow{6}{*}{ fast } & \multirow{3}{*}{ small } & \multirow{3}{*}{1} & 2 & -0.07 & 0.41 & 44 \\
\hline & & & 2.5 & -0.08 & 0.26 & 50 \\
\hline & & & 3 & -0.26 & 0.28 & 75 \\
\hline & \multirow{3}{*}{ big } & \multirow{3}{*}{1.5} & 2 & -0.31 & 0.46 & 62 \\
\hline & & & 2.5 & -0.58 & 0.47 & 81 \\
\hline & & & 3 & -0.85 & 0.36 & 94 \\
\hline \multirow{6}{*}{ slow } & \multirow{3}{*}{ small } & \multirow{3}{*}{2} & 2 & -0.69 & 0.67 & 70 \\
\hline & & & 2.5 & -0.90 & 0.64 & 88 \\
\hline & & & 3 & -1.00 & 0.39 & 86 \\
\hline & \multirow{3}{*}{ big } & \multirow{3}{*}{3} & 2 & -1.20 & 0.75 & 80 \\
\hline & & & 2.5 & -1.13 & 1.21 & 69 \\
\hline & & & 3 & -1.81 & 1.14 & 86 \\
\hline
\end{tabular}

Note. $M=$ mean, $S D=$ standard deviation.

${ }^{1}$ A trial was defined as anticipatory if at least one raw gaze position (blinks and saccades removed) was on the reappearance AOI during the full occlusion. 


\section{Figure Captions}

Figure 1. Exemplary stills of the continuous motions presented in the eye-tracking task (panel A); areas of interest (AOIs) that were used to analyze gaze positions in the eyetracking tasks (panel B); stimuli presented in the wooden blocks tasks, with order indicated by arrows (panel C); example of a trial sequence in the wooden block task (panel D).

Figure 2. Percentage of trials the toddlers looked at a specific area of interest (AOI) in the eye-tracking task, averaged across toddlers. Time windows range from the start of the animation until $1.5 \mathrm{~s}$ after the end of the full occlusion. The lines were slightly smoothed with the LOESS function.

Figure 3. Eye-tracking measures by age. (A) Switches from stimulus to pivot AOI during visible rotation. (B) Percentage of time the toddlers looked at the stimulus AOI during visible rotation. (C) Arrival time at the reappearance AOI. (D) Looking back to the disappearance AOI. Columns show results from fast and slow velocity conditions.

Figure 4. Results of the wooden block task. (A) Age-related changes in preadjustment. (B) Percent of the trials solved by time elapsed. Note that at the hard difficulty, age differences were present early on and persisted. (C) Age-related changes in success within $4 \mathrm{~s}$ since trial start. (D) Percentage of the trials solved successfully (i.e., within the available 20 s) by age and difficulty level. The lines in (C) and (D) are LOESS curves.

Figure 5. Correlation between arrival time in the eye-tracking task and preadjustment in the wooden block task by age and velocity. 

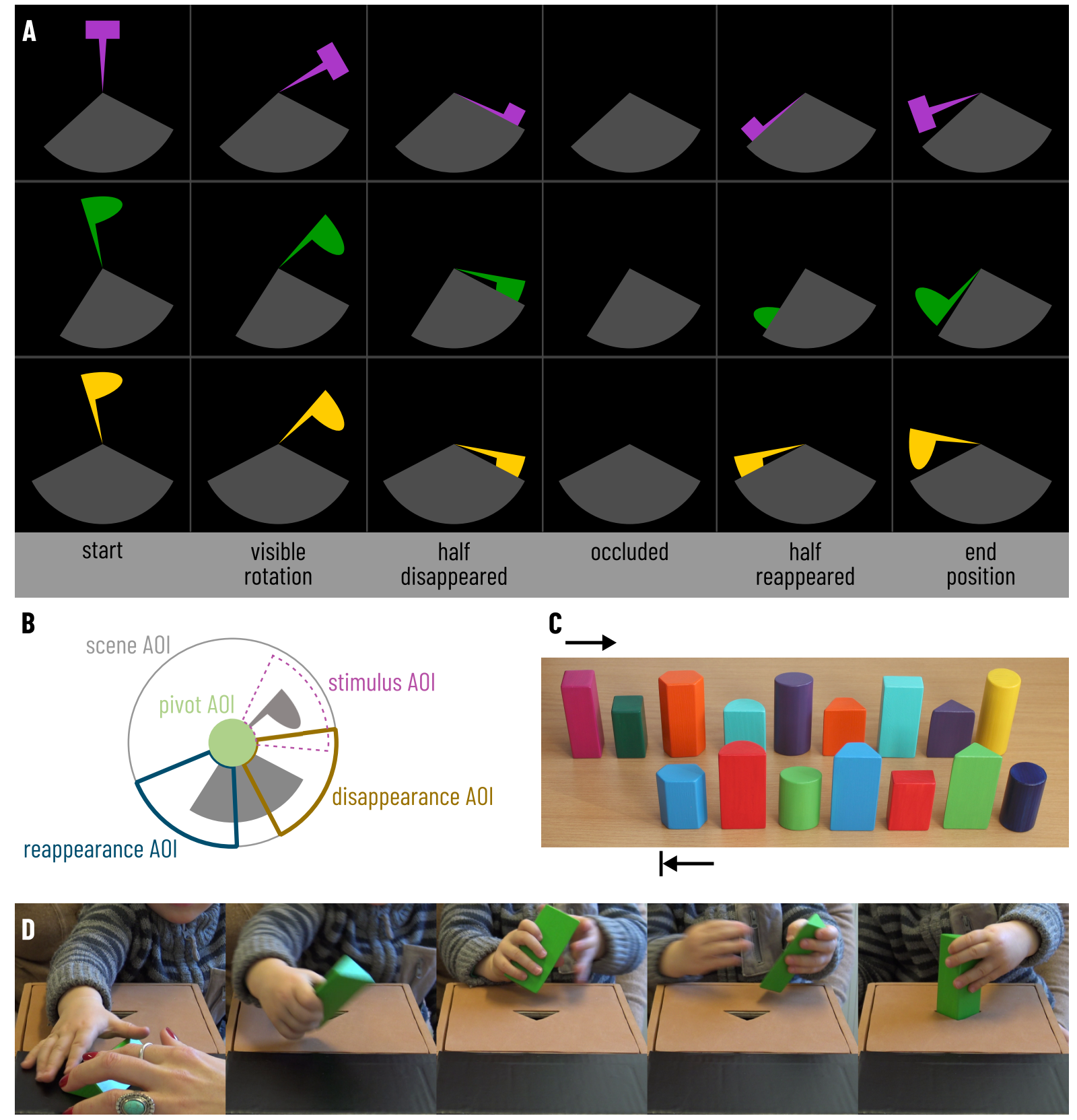

Figure 1 
small occluder
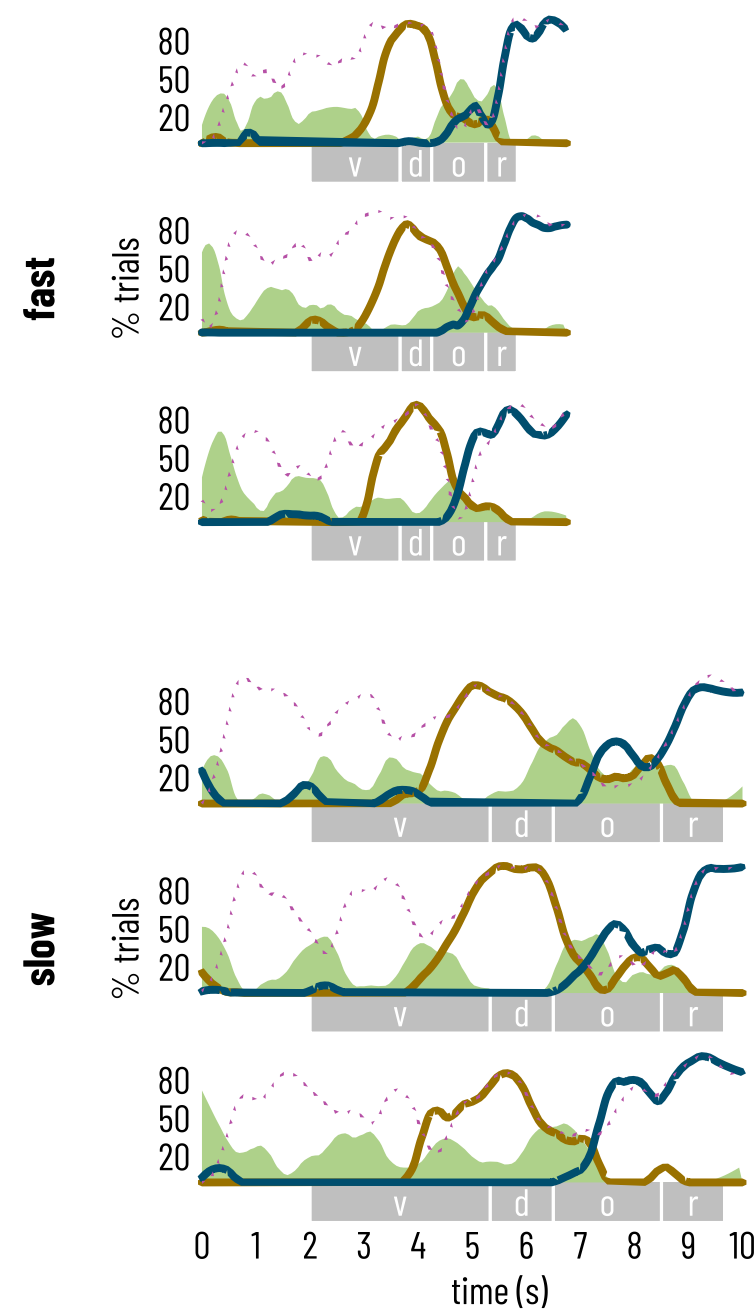

\section{big occluder}
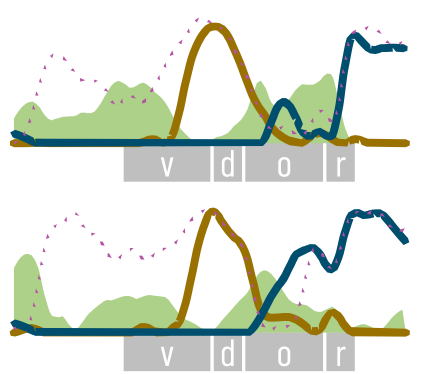

2.5 years

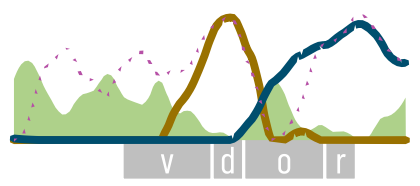

3 years

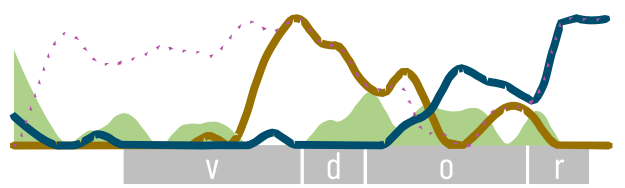

2 years

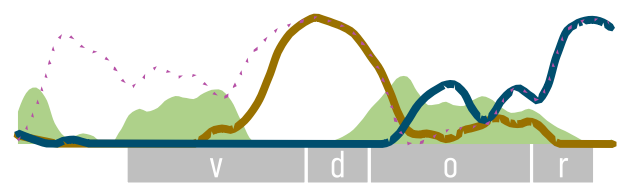

2.5 years

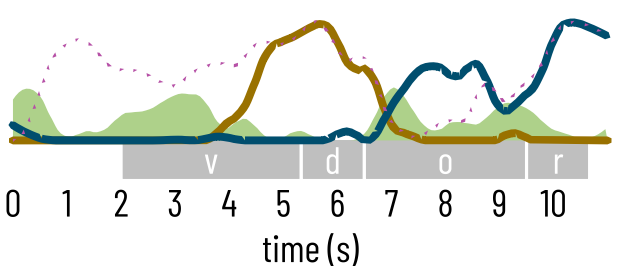

2 years

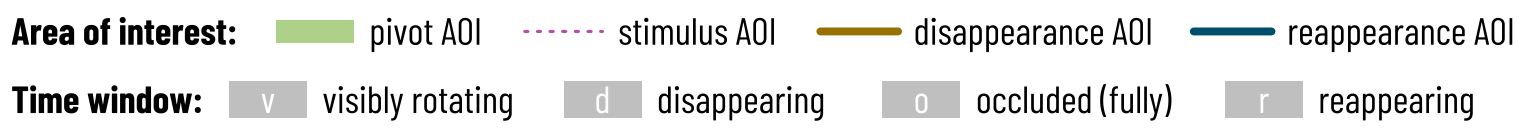

Figure 2 


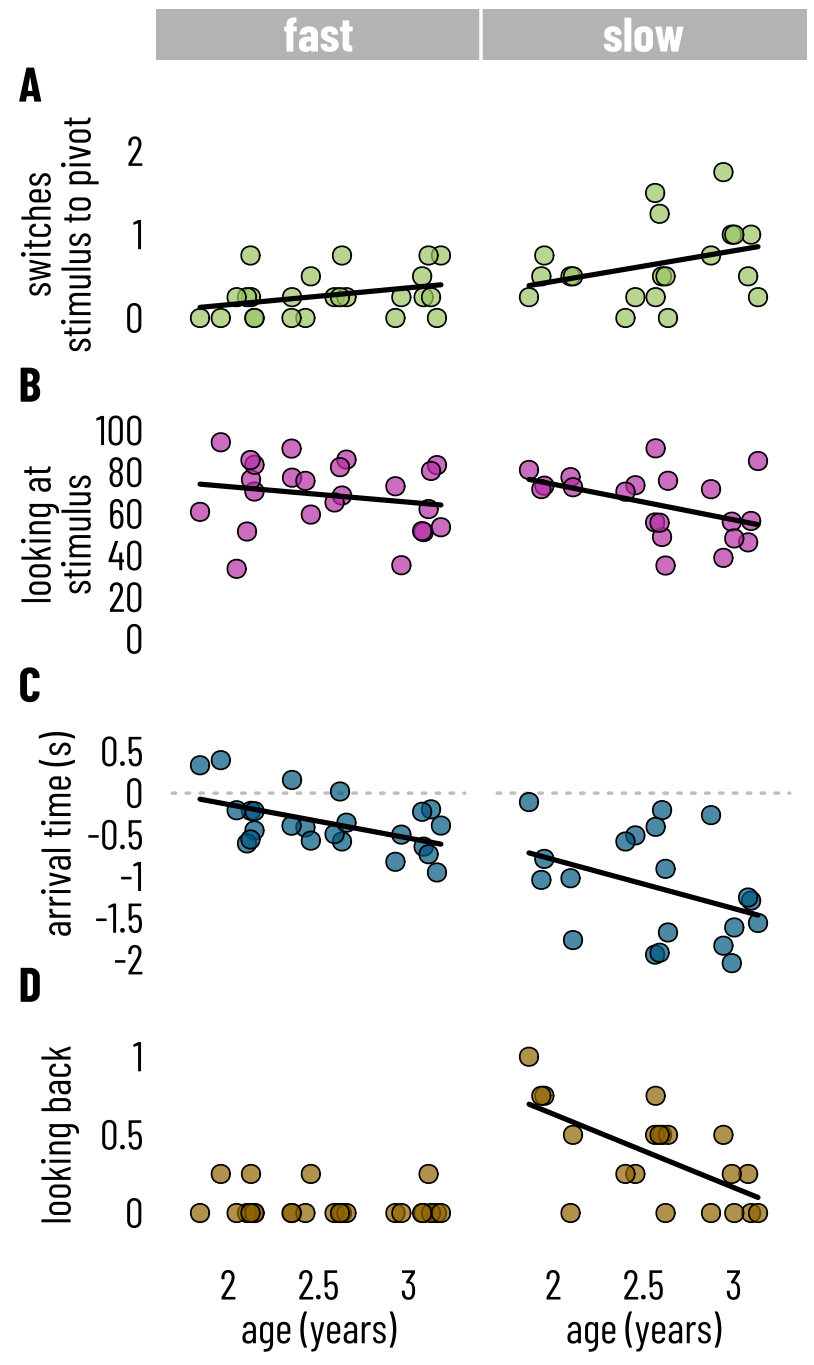

Figure 3 


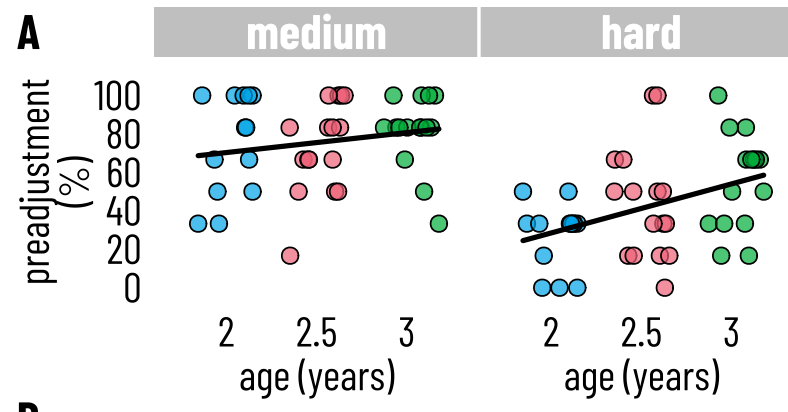

B

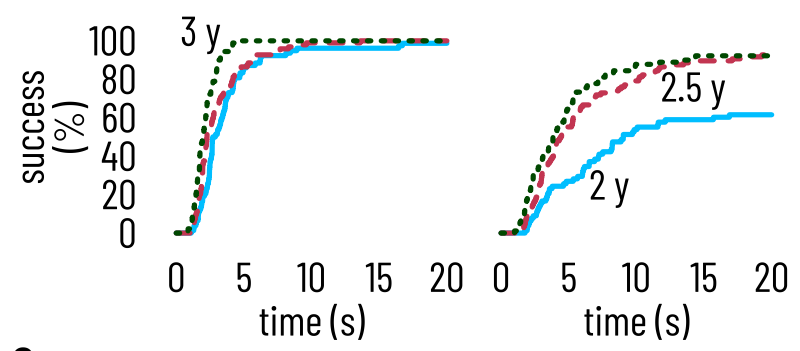

C

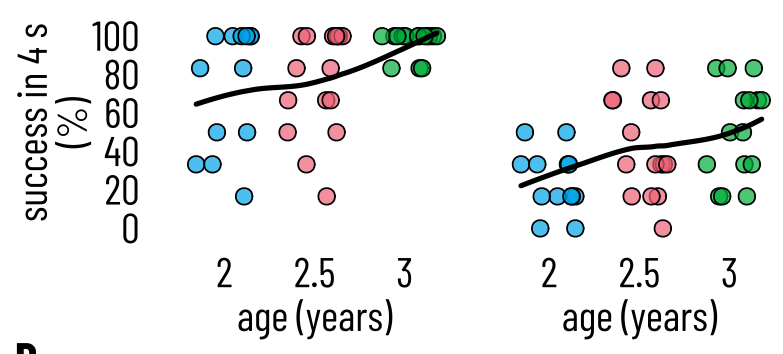

D

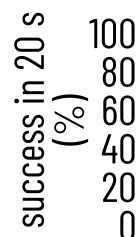

c000

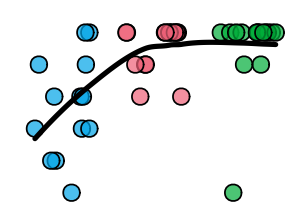

$2.5 \quad 3$
age (years)

2.5
age (years)

Figure 4 


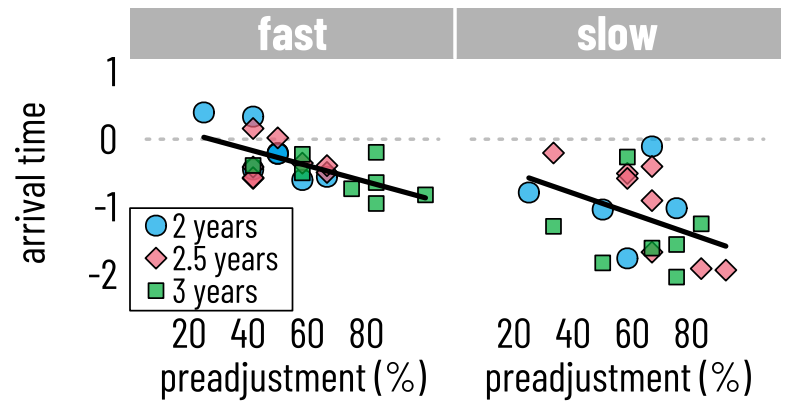

Figure 5 